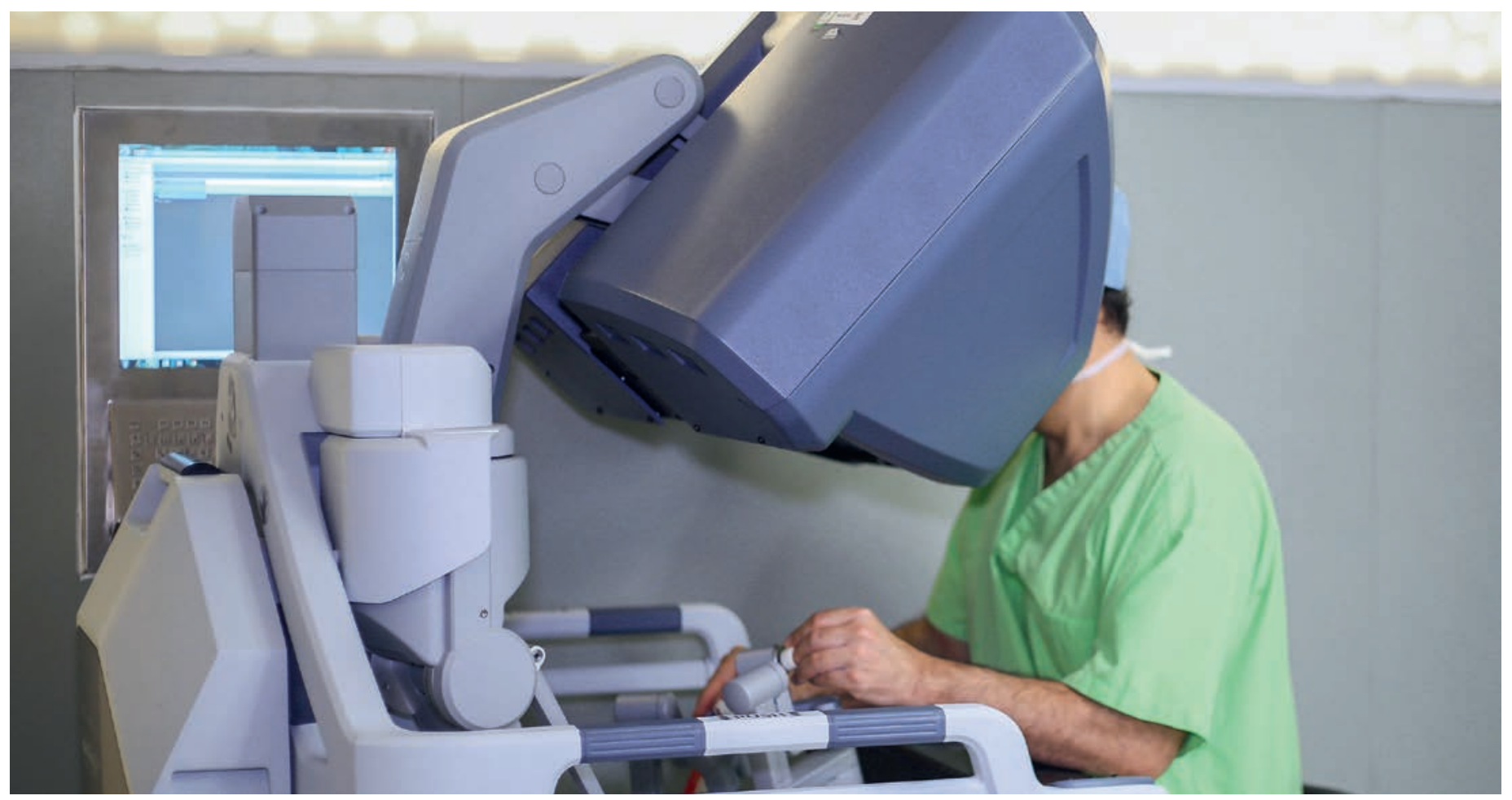

\title{
Roboterassistierte Operationen nur in Ausnahmefällen sinnvoll
}

\section{Susanna Marti Calmell}

Trägerschaft Swiss Medical Board

In seinem neuesten Bericht vergleicht das Swiss Medical Board (SMB) roboterassistierte und konventionelle Operationsverfahren bei radikaler Prostatektomie und bei Hysterektomie. Dabei fand sich keine überzeugende Evidenz, welche die Bevorzugung der roboterassistierten Verfahren rechtfertigen würde. Dies trifft für die einfache und radikale Hysterektomie und für die radikale Prostatektomie zu, namentlich für Spitäler mit geringen Fallzahlen.

Prostata- und Gebärmutterkarzinome sind in der Schweiz verbreitete Erkrankungen und häufige Ursachen für krebsbedingte Todesfälle. In beiden Fällen sind die offene Operation oder ein minimal-invasiver

\section{Im Juli 2018 waren in Schweizer Spitälern insgesamt 33 Robotersysteme im Einsatz.}

laparoskopischer Eingriff (mit oder ohne Unterstützung durch ein Robotersystem) gängige Behandlungs- optionen. Im Juli 2018 waren in Schweizer Spitälern insgesamt 33 Robotersysteme im Einsatz; bei allen handelte es sich um DaVinci ${ }^{\circledR}$-Roboter, deren Kaufpreis bei etwa 1,8 Millionen CHF pro System liegt. Der aktuelle SMB-Bericht vergleicht nun Wirksamkeit, Sicherheit und Kosten-Nutzen-Verhältnis des roboterassistierten Verfahrens mit dem jeweils herkömmlichen Operationsverfahren d.h. der offenen radikalen Prostatektomie bzw. der konventionellen laparoskopischen Hysterektomie. 
Für die Bewertung der Wirksamkeit und der Sicherheit der unterschiedlichen Operationsverfahren stützt sich der Bericht auf die Ergebnisse von randomisierten klinischen Studien, die in zwei Cochrane Reviews zusammengefasst wurden. Für die gesundheitsökonomische Analyse wurden relevante internationale Studien mit einer neuen Kostenanalyse ergänzt; diese verwendete Schätzungen aus der Schweiz sowie Daten von

\section{Aus Sicht des SMB favorisiert die verfügbare klinische Evidenz weder die roboterassistierte noch die konventionelle laparoskopische Hysterektomie.}

Schweizer Krankenversicherern. Die Gesamtbewertung beruht auf dem «Evidence to Decision»-Rahmenwerk von GRADE.

Die Resultate der roboterassistierten radikalen Prostatektomie unterscheiden sich in Bezug auf erwünschte Wirkungen (z.B. Blasen- und sexuelle Funktion) nur wenig von der offenen radikalen Prostatektomie, während die Unterschiede z. B. in Bezug auf eine Reduktion der postoperativen Schmerzen als mässig zu beurteilen sind. In der Kostenanalyse fielen bei Patienten mit roboterassistierter radikaler Prostatektomie höhere Kosten an (ca. 4000 CHF). Allerdings gibt es kaum zuverlässige Kostenschätzungen für die Routineversorgung in Schweizer Spitälern. Dies schränkt die Gültigkeit der gesundheitsökonomischen Analyse ein. Der zusätzliche Ressourcenbedarf für die roboterassistierte radikale Prostatektomie im Vergleich zur offenen radikalen Prostatektomie wird als moderat eingestuft; dieser wäre noch niedriger, wenn sich deren Einsatz auf wenige Zentren beschränken und somit zu höheren Fallzahlen pro Robotersystem führen würde. Die vorhandene Evidenz spricht gegen die Anwendung

\section{Die Entscheidung der Patienten fiele wahr-} scheinlich anders aus, wären sie über die Operationsverfahren umfassend informiert worden.

von roboterassistierter radikaler Prostatektomie in Spitälern mit geringer Fallzahl.

Auch bei der roboterassistierten Hysterektomie sind die Unterschiede in Bezug auf die erwünschten Wirkungen gegenüber der konventionellen laparoskopischen Hysterektomie unwesentlich, und die unerwünschten Wirkungen sind bei beiden chirurgischen Verfahren vergleichbar. Aus Sicht des SMB favorisiert die verfügbare klinische Evidenz weder die roboterassistierte noch die konventionelle laparoskopische Hysterektomie. In der Kostenanalyse übertreffen die
Kosten pro roboterassistierter Hysterektomie bei gutartigen Erkrankungen die mit dem konventionellen laparoskopischen Eingriff verbundenen Kosten um ca. 5500 CHF. Bei bösartigen Erkrankungen wie Gebärmutterhals- oder Eierstockkarzinomen übersteigen die Kosten der roboterassistierten Hysterektomie jene der konventionellen laparoskopischen Hysterektomie um ca. 4300 CHF. Dieser zusätzliche Ressourcenbedarf wird als moderat eingestuft. Aus Sicht des SMB spricht das Kosten-Nutzen-Verhältnis sowie das Fehlen von zuverlässigen Daten, die einen medizinischen Mehrwert einer roboterassistierten Hysterektomie untermauern, zugunsten einer Anwendung des konventionellen laparoskopischen Verfahrens bei der einfachen oder radikalen Hysterektomie.

Eine Analyse der publizierten Evidenz bezüglich Patientenpräferenzen, Chancengleichheit im Gesundheitswesen oder Akzeptanz der roboterassistierten Technologie durch die Patienten war nicht Teil des Assessment-Berichts. Das SMB stellt in Frage, ob die gegenwärtige Praxis, wie Patienten über die verfügbaren Behandlungsoptionen informiert werden, eine ausreichende Grundlage für deren Einwilligung für eine bestimmte Eingriffsart bildet. Die Entscheidung der Patienten fiele wahrscheinlich anders aus, wären sie über die Operationsverfahren umfassend informiert worden.

Der vollständige Bericht kann auf der Webseite des Swiss Medical Board eingesehen werden unter https://www.swissmedicalboard.ch

\section{Bildnachweis}

(c) Oleksandr Kontsevoi | Dreamstime.com (Symbolbild)

\section{Informationen zum SMB}

Zwei Pioniere des SMB sind nach 8 Jahren Amtszeit zurückgetreten: Peter Suter als Präsident desTrägervereins und Urs Metz ger als Vorsitzender des Appraisal-Komitees. Daniel Scheidegger, Präsident SAMW, wurde zum Nachfolger von Peter Suter ernannt. Christoph A. Meier, CMO des Unispitals Basel, hat die Nachfolge von Urs Metzger angetreten.

Zudem wurde die Trägerschaft erweitert: Neu sind der Verband chirurgisch und invasiv tätiger Fachgesellschaften Schweiz (FMCH) und die Medizinaltarif-Kommission UVG (MTK) Mitglieder des Trägervereins SMB.

Die Geschäftsstelle des SMB zieht von Zürich nach Bern. Die neue Postadresse lautet: Swiss Medical Board, Haus der Akademien, Laupenstrasse 7, 3001 Bern. Tel.: +4176 5150220 